\title{
A retrospective analysis of geriatric trauma patients: venous lactate is a better predictor of mortality than traditional vital signs
}

\author{
Kristin M Salottolo ${ }^{1,2}$, Charles W Mains ${ }^{1,3,4}$, Patrick J Offner ${ }^{1,3,4}$, Pamela W Bourg ${ }^{3}$ and David Bar-Or $r^{1,2,4^{*}}$
}

\begin{abstract}
Background: Traditional vital signs (TVS), including systolic blood pressure (SBP), heart rate (HR) and their composite, the shock index, may be poor prognostic indicators in geriatric trauma patients. The purpose of this study is to determine whether lactate predicts mortality better than TVS.

Methods: We studied a large cohort of trauma patients age $\geq 65$ years admitted to a level 1 trauma center from 2009-01-01 - 2011-12-31. We defined abnormal TVS as hypotension (SBP $<90 \mathrm{~mm} \mathrm{Hg}$ ) and/or tachycardia ( $H R>120$ beats/min), an elevated shock index as HR/SBP $\geq 1$, an elevated venous lactate as $\geq 2.5 \mathrm{mM}$, and occult hypoperfusion as elevated lactate with normal TVS. The association between these variables and in-hospital mortality was compared using Chi-square tests and multivariate logistic regression.

Results: There were 1987 geriatric trauma patients included, with an overall mortality of $4.23 \%$ and an incidence of occult hypoperfusion of $20.03 \%$. After adjustment for GCS, ISS, and advanced age, venous lactate significantly predicted mortality (OR: 2.62, $p<0.001$ ), whereas abnormal TVS (OR: 1.71, $p=0.21$ ) and $S I \geq 1$ (OR: 1.18, $p=0.78$ ) did not. Mortality was significantly greater in patients with occult hypoperfusion compared to patients with no sign of circulatory hemodynamic instability $(10.67 \%$ versus $3.67 \%, p<0.001)$, which continued after adjustment (OR: 2.12 , $p=0.01$.

Conclusions: Our findings demonstrate that occult hypoperfusion was exceedingly common in geriatric trauma patients, and was associated with a two-fold increased odds of mortality. Venous lactate should be measured for all geriatric trauma patients to improve the identification of hemodynamic instability and optimize resuscitative efforts.
\end{abstract}

Keywords: Geriatric, Occult hypoperfusion, Shock index, Circulatory hemodynamic instability

\section{Background}

Initial triage, trauma team activation, and early resuscitation of trauma patients is often directed by the presence of abnormal traditional vital signs (TVS) such as systolic blood pressure (SBP) and heart rate (HR), as these measures are physical signs of circulatory hemodynamic instability (cHI). The shock index (SI), a composite of TVS, may be a more sensitive and accurate predictor of hypoperfusion and early shock than its individual

\footnotetext{
* Correspondence: dbaror@ampiopharma.com

${ }^{1}$ Trauma Research Department, St. Anthony Hospital, 11600 W. 2nd Place, Lakewood, CO 80228, USA

${ }^{2}$ Trauma Research Department, Swedish Medical Center, Englewood, CO 80113, USA

Full list of author information is available at the end of the article
}

components $[1,2]$. However, these markers may not be a sign of cHI in all patients following traumatic injury [3].

Geriatric patients respond to trauma and shock differently than their younger counterparts due to the presence of comorbid conditions, decreased physiologic reserve, elasticity of the vascular system, and concomitant medication use. Decreased physiologic reserve reduces the ability to respond to injury or tolerate aggressive resuscitation [4], while polypharmacy can alter the hemodynamic response to shock and complicate the patient's clinical picture [5]. As a result, the severity of injury and the response to resuscitative efforts are often underestimated because the absence of tachycardia and hypotension can be misleading [6,7]. Martin et al. demonstrated that current triage criteria using SBP and 
HR are inadequate when applied to elderly trauma patients [7], leading to frequent undertriage in the elderly with a high risk of death [8].

Hypoperfusion is associated with the failure to maintain capillary oxygen delivery, leading to anaerobiosis and lactate production in excess to its rate of metabolism. The amount of lactate produced is believed to correlate with the total oxygen debt, the magnitude of hypoperfusion, and the severity of shock. Venous lactate $(\mathrm{VL})$ is an accessible, accurate, validated measurement, and the measurement of admission lactate level may facilitate the recognition of occult hypoperfusion $(\mathrm{OH})$ and shock [9-11], as VL is a metabolic indicator of cHI.

Elevated lactate levels have been consistently shown as a marker of severe injury and mortality in trauma patients, particularly in patients older than 55 years [10-16]. Despite this knowledge, few activation and resuscitation guidelines include $\mathrm{VL}$ as a measure of $\mathrm{cHI}$, rather focusing on TVS like SBP and HR [17-19]. We instituted a geriatric resuscitation protocol in 2009 that includes a VL measurement for all geriatric trauma patients upon presentation to the emergency department (ED), and resuscitation based on VL values [20]. Prior to this new geriatric protocol, activation and resuscitation at our institution was based on mechanism, physical findings, Glasgow Coma Scale (GCS) and abnormal TVS. With advances in healthcare resulting in longer and healthier lifestyles in the elderly [21], there is greater potential for traumatic injury. Identifying an optimal marker of $\mathrm{cHI}$ has implications for initial assessment and resuscitation protocols for this ever increasing geriatric trauma population. We hypothesized that VL would have better predictive ability for mortality than SBP, HR, or SI in geriatric trauma patients.

\section{Methods}

\section{Patients and setting}

This retrospective cohort study evaluated the medical records of all consecutively admitted geriatric (age $\geq 65$ ) trauma patients from January 1st, 2009 through

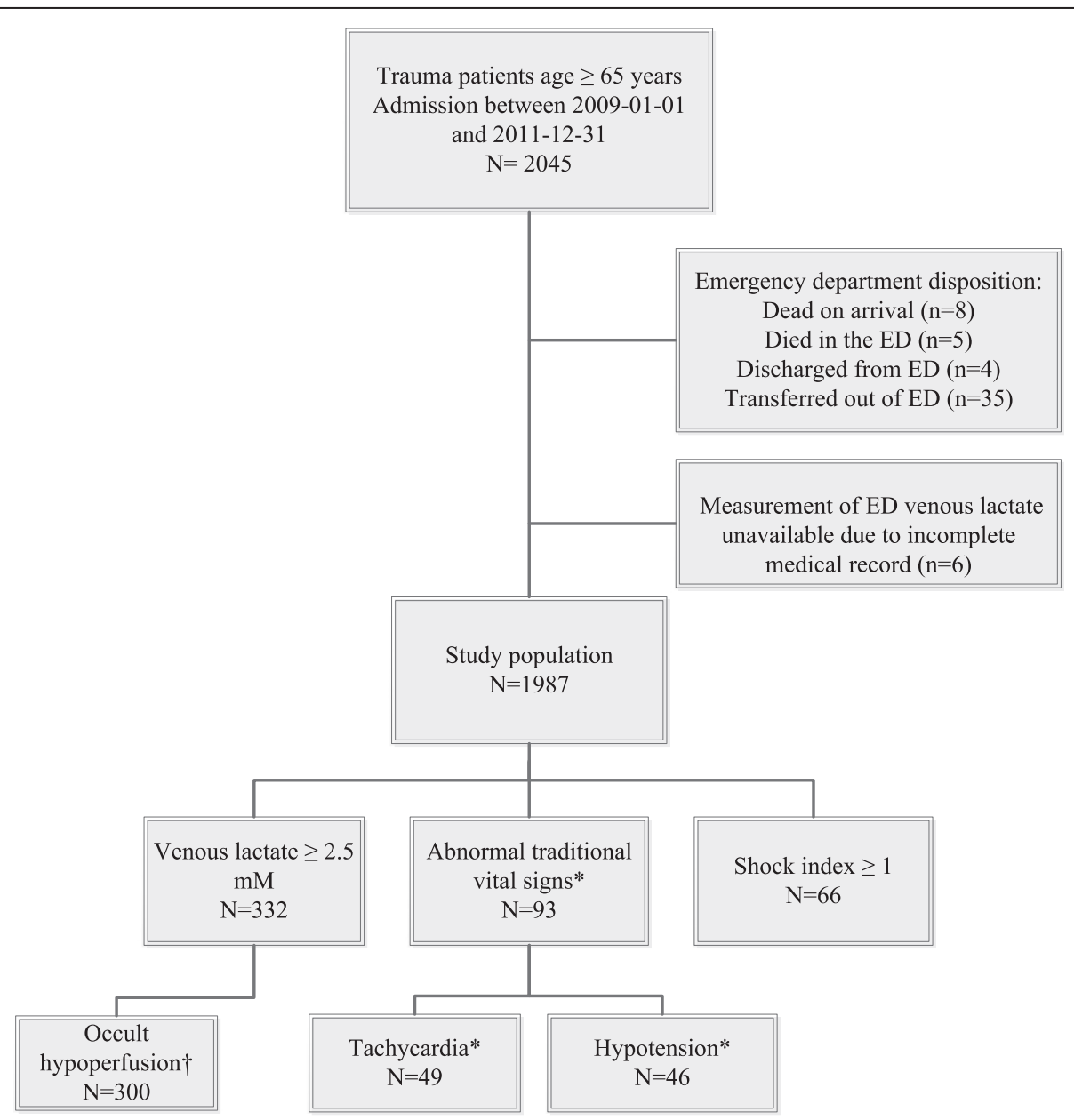

Figure 1 Patient disposition, by physiologic variable of interest. *Abnormal traditional vital signs: Hypotension (systolic blood pressure $<90 \mathrm{~mm} \mathrm{Hg}$ ) and/or tachycardia (heart rate $>120$ beats/min). †Occult hypoperfusion: Venous lactate $\geq 2.5 \mathrm{mM}$ with normal traditional vital signs. 
December 31st, 2011 to St. Anthony Hospital, an urban Level I Trauma Center located in Lakewood, Colorado. A trauma patient is defined according to a principal diagnosis of trauma based on ICD-9-CM diagnostic injury codes. Additional selection criteria can be found in the State registry manual [22]. Patients were excluded based on ED disposition, as follows: dead on arrival $(\mathrm{n}=8)$, died in the ED $(\mathrm{n}=5)$, discharged from the ED $(\mathrm{n}=4)$ or transferred-out of the ED $(\mathrm{n}=35$, including transfers to pediatric trauma centers, burn centers, or Kaiser Permanente medical facilities), Figure 1. We also excluded patients for whom we were unable to determine if a VL had been drawn due to incomplete medical records $(n=6)$. Dedicated trauma registrars prospectively abstracted data on all trauma admissions into our trauma registry (TraumaBase $\odot$, Conifer $\mathrm{CO}$ ).

\section{$\mathrm{cHI}$ measurements}

Vital signs, including SBP and HR measurements, were taken manually upon arrival to the ED, followed by automated measurements whose frequency was based on the patient's acuity. After initial vital signs were measured, VL measurements were performed as follows: an anaerobic blood sample was obtained from an upper extremity peripheral venipuncture, or occasionally from a central line if one was placed emergently, and analyzed with the ABL-700 blood gas analyzer (Radiometer Analytical, Copenhagen Denmark). If the patient presented with an elevated VL, interventions included volume resuscitation and enhanced vigilance. If persistent, then central venous monitoring was performed if not already established.

\section{Outcome evaluation}

The primary outcome was in-hospital mortality. The following variables were analyzed for their association with and predictive capability for in-hospital mortality: elevated VL $(\geq 2.5 \mathrm{mM})$, hypotension $(\mathrm{SBP}<90 \mathrm{~mm}$ $\mathrm{Hg}$ ), tachycardia (elevated HR $>120$ beats/minute), abnormal TVS (the presence of hypotension and/or tachycardia), and elevated shock index $\geq 1$. An elevated VL in the $E D \geq 2.5 \mathrm{mM}$ was used for this analysis based on previous studies that identified an increased risk of mortality with lactate $\geq 2.5 \mathrm{mM}$ [23-25], and based on the geriatric resuscitation protocol's VL parameters for initiating resuscitation protocols. We also examined mortality related to $\mathrm{OH}$, defined as elevated $\mathrm{VL}$ in the presence of normal TVS.

Covariates included age, sex, ISS, GCS, mechanism of injury, respiratory rate, and admission service. These variables were categorized as follows: age (65-84 and $\geq$ 85 years), sex (male and female), ISS $(\geq 16$ and $<16$ ), GCS (3-12 and 13-15), respiratory rate $(<10$ or $>29$ breaths/minute and 10-29), cause of injury (fall, motor vehicle accident, and all other types), and admission service (trauma service and all other services).

\section{Statistical analysis}

Statistical analyses were performed using SAS ${ }^{\circledR}$ software, version 9.2 (SAS Institute, Cary, NC). We examined inhospital mortality using Pearson and Fisher's exact chisquare tests and multivariate logistic regression. Stepwise multivariate logistic regression models were used to examine whether the presence of the physiologic variables of interest (elevated VL, SI, HR, and SBP, abnormal TVS, and $\mathrm{OH}$ ) independently predicted inhospital mortality. All covariates that met entry and exit values of $\mathrm{p}<0.15$ were included in the stepwise logistic regression models, independent of whether they were significant predictors of mortality in univariate analyses. Each logistic regression model was built using separate stepwise selection; however, all models found the following variables to be significant at entry and exit values of $p<0.15$ : GCS, ISS and age. Adjusted Rsquared and Hosmer-Lemoshow goodness of fit statistics were examined to assess how well the models fit the data; lower R-squared values and smaller p-values indicate a lack of fit of the model. Data are presented as odds ratios (OR) with $95 \%$ confidence intervals (CI), as well as medians with interquartile range. For all analyses, significance was set at $\mathrm{p}$-value $<0.05$. This study was approved by the St. Anthony Hospital Institutional Review Board.

\section{Results}

\section{Patient demographics}

There were 1987 geriatric trauma patients included in the study (Figure 1). The median age was 79 (72-86) years, and fall cause of injury occurred in $78.16 \%$ of patients. The most frequent service for admission was trauma surgery $(55.11 \%)$, nonsurgical (e.g. critical care, medicine; 39.31\%) and orthopedic services (4.78\%). Overall injury severity was low with a median ISS of 9 (4-10) and median ED GCS of 15 (15-15). Despite the relatively minor injuries, the median length of stay was 4 (2-6) days, and the most common discharge disposition was to a skilled nursing facility (43.35\%).

Ninety-three patients $(4.72 \%)$ presented with abnormal TVS, and 66 patients (3.38\%) presented with a SI $\geq 1$, Figure 1. Of the 93 patients with abnormal TVS, 32 patients $(39.02 \%)$ also had a known elevated VL. There were 1498 patients (75.39\%) who had an ED VL drawn; of those, 332 patients (22.16\%) had an elevated VL. Thirty-two patients $(9.64 \%)$ with an elevated VL also presented with abnormal TVS; the remaining 300 patients $(90.36 \%)$ presented with $\mathrm{OH}$. Differences in clinical and demographic characteristics between 
patients with abnormal TVS and elevated VL are presented in Table 1.

\section{Mortality}

There were 84 patients who expired in the hospital (4.23\%); approximately half of all deaths were patients with an elevated lactate $(52.63 \%, 40 / 76$ patients with a known lactate), while only 13 patients who expired presented with abnormal TVS (15.48\%, 13/84).

As expected, mortality was significantly greater for patients with severe overall injuries (ISS $\geq 16$ and $<16: 16.00 \%$ vs. $2.26 \%$, respectively, $\mathrm{p}<0.001$ ), moderate to severe GCS (3-12 and $13-15$ : $36.97 \%$ vs. $2.12 \%$, respectively, $\mathrm{p}<0.001$ ), and abnormal ED respiratory rate $(<10$ or $>29$ and 10-29: $24.00 \%$ vs. $3.79 \%$, respectively, $\mathrm{p}<0.001)$. Mortality was also significantly greater in males than females $(5.51 \%$ and $3.15 \%$, respectively, $\mathrm{p}=0.009$ ), and admission to the trauma service than all other services $(5.39 \%$ and $2.80 \%$, respectively, $\mathrm{p}=0.004)$. There were no differences in mortality according to age (65-84 and $\geq 85: 3.84 \%$ and $5.18 \%$, respectively, $\mathrm{p}=0.18)$, and cause of injury ( $3.73 \%$ falls, $5.45 \%$ motor vehicle accident, $6.78 \%$ all other causes, $\mathrm{p}=0.09$ ).

Before adjustment, mortality was significantly different in patients with abnormal vs. normal TVS, SBP, HR, and SI (Table 2). However, after adjustment for ISS, GCS, and advanced age, mortality was not significantly higher in patients with abnormal TVS or SI $\geq 1$ (Table 3), nor were the individual components significant: $\mathrm{SBP}<90$ mm Hg (OR: 1.93 [0.65 - 5.70], $\mathrm{p}=0.24$ ) and HR > 120 beats/min (OR: 1.41 [0.41 - 4.79], $\mathrm{p}=0.58$ ), data not shown.

Conversely, an elevated VL $\geq 2.5 \mathrm{mM}$ with or without abnormal TVS was significantly associated with mortality both prior to and after adjustment for ISS, GCS, and advanced age (Table 3). There was a 2.62-fold increased odds of mortality in patients with a $\mathrm{VL} \geq 2.5 \mathrm{mM}$, which persisted when patients with $\mathrm{OH}$ were analyzed.

When a multivariate regression incorporating both VL and TVS was performed, elevated VL continued to predict mortality (OR: 2.58, p < 0.001), whereas abnormal TVS was not predictive of mortality (OR: $1.46, \mathrm{p}=0.39$ ), Table 3.

A moderate-to-severe GCS (3-12) was the most predictive of mortality in all four models, followed by overall injury severity (ISS $\geq 16$ ); the best fitting model incorporated VL. Although the model's estimates fit the data at an acceptable level according to the HosmerLemoshow goodness of fit test, just $30 \%$ of the variability in mortality can be explained by the explanatory variables. The remaining $70 \%$ of the variability in

Table 1 Demographic and clinical characteristics by presence of abnormal traditional vital signs (TVS) and elevated venous lactate*

\begin{tabular}{|c|c|c|c|}
\hline Variable, \% (n) & Abnormal TVS $(n=93) \dagger$ & Lactate $\geq 2.5 \mathrm{mM}(\mathrm{n}=332)$ & missing (n) \\
\hline Abnormal TVSt & $100(93)$ & $9.64(32)$ & 16 \\
\hline Lactate $\geq 2.5 \mathrm{mM}$ & $39.02(32)$ & 100 (332) & 489 \\
\hline Mortality & $13.98(13)$ & $12.05(40)$ & 0 \\
\hline Blunt mechanism & $96.74(89)$ & $97.89(325)$ & 10 \\
\hline Female gender & $49.46(46)$ & $48.49(161)$ & 0 \\
\hline Advanced age $\geq 85$ & $20.43(19)$ & $25.30(84)$ & 0 \\
\hline$I S S \geq 16$ & $38.89(35)$ & $27.58(91)$ & 29 \\
\hline GCS $3-8$ & $22.58(21)$ & $15.06(50)$ & 27 \\
\hline Respiratory rate $<10$ or $>29$ & $6.82(6)$ & $3.79(12)$ & 62 \\
\hline Trauma team activation & $53.76(50)$ & $40.66(135)$ & 0 \\
\hline Cause & & & 0 \\
\hline Fall & $65.59(61)$ & $64.16(213)$ & \\
\hline Motor vehicle & $23.66(22)$ & $23.49(78)$ & \\
\hline ED disposition: & & & 7 \\
\hline Intensive care unit & $37.63(35)$ & $47.29(157)$ & \\
\hline Floor & $31.18(29)$ & $35.24(117)$ & \\
\hline Discharge disposition: & & & 1 \\
\hline LTAC/SNF/rehabilitation & $65.59(61)$ & $46.69(155)$ & \\
\hline Home & $17.20(16)$ & $33.73(112)$ & \\
\hline
\end{tabular}

ISS, injury severity score; GCS, glasgow coma scale; ED, emergency department; LTAC, long term acute care; SNF, skilled nursing facility.

*There were 11 patients who had both abnormal TVS and elevated venous lactate.

†Abnormal TVS: Systolic blood pressure $<90 \mathrm{~mm} \mathrm{Hg}$ and/or heart rate $>120$ beats $/ \mathrm{min}$. 
Table 2 Comparison of in-hospital mortality rates, by presence of abnormal circulatory hemodynamic instability marker

\begin{tabular}{lccc}
\hline \multirow{2}{*}{ Marker } & \multicolumn{2}{c}{ Mortality \% (n) } & \\
\cline { 2 - 3 } & Abnormal & Normal & P value \\
\hline Venous lactate & $12.05(40 / 332) *$ & $3.09(36 / 1166)$ & $<\mathbf{0 . 0 0 1}$ \\
Traditional vital sign & $13.98(13 / 93) \dagger$ & $3.78(71 / 1878)$ & $<\mathbf{0 . 0 0 1}$ \\
Systolic blood pressure & $19.57(9 / 46) \neq$ & $3.85(74 / 1920)$ & $<\mathbf{0 . 0 0 1}$ \\
Heart rate & $12.24(6 / 49) \S$ & $3.98(76 / 1908)$ & $\mathbf{0 . 0 1}$ \\
Shock index & $10.61(7 / 66) \uparrow$ & $3.82(72 / 1884)$ & $\mathbf{0 . 0 2}$ \\
\hline
\end{tabular}

*Abnormal venous lactate: $\geq 2.5 \mathrm{mM}$.

tAbnormal TVS: Systolic blood pressure $<90 \mathrm{~mm} \mathrm{Hg}$ and/or heart rate $>$ 120 beats/min.

$\ddagger$ Abnormal systolic blood pressure: $<90 \mathrm{~mm} \mathrm{Hg}$

$\S$ Abnormal heart rate: $>120$ beats/min

१Abnormal shock index: $\geq 1$.

mortality is explained by either inherent variability or variables not included in the models. Logistic regression models that included all variables with $\mathrm{p}<0.15$ in univariate analyses produced slightly lower R-squared values than the stepwise models presented in Table 3.

\section{Discussion}

Guidelines for initial triage, trauma team activation, and early resuscitation following trauma are largely based on studies performed in younger trauma patients [26]. The geriatric population appears to be a relevant subset of patients in whom traditional physical signs of $\mathrm{cHI}$ such as SBP and HR may not identify severe injury and blood loss [6,7], and in whom additional indicators of $\mathrm{cHI}$ such as VL are important in predicting mortality. The results of this study demonstrate that a VL $\geq 2.5 \mathrm{mM}$ was independently associated with a 2.6 -fold increased odds of mortality in geriatric trauma patients. The TVS of SBP, $\mathrm{HR}$, and SI were not independently associated with mortality in geriatric trauma patients, and activation based on abnormal TVS does not appear to independently identify a population of patients at risk of mortality. These results were demonstrated in a high volume level I trauma center with a large percentage of elderly trauma patients.

Perhaps more importantly, $\mathrm{OH}$ was exceedingly common in elderly trauma patients, occurring in $20.03 \%$, and was associated with a two-fold increased odds of mortality. There continues to be a high frequency of $\mathrm{OH}$ ranging from $16 \%$ to $70 \%$ in hemodynamically stable severely injured patients [27-29]. At our institution we identified elderly trauma patients who appeared to be hemodynamically stable in the ED, and would later deteriorate in the operating room or in a non-intensive care unit setting because they were not adequately resuscitated or triaged. A protocol was implemented that utilizes VL on admission, and sets in motion a resuscitative protocol for patients with a $\mathrm{VL} \geq 2.5 \mathrm{mM}$. It is important for inadequate perfusion to be recognized during initial resuscitation efforts, because prolonged $\mathrm{OH}$ in elderly trauma increases mortality from $12 \%$ to $35 \%$ [13]. A cut-off for $\mathrm{VL}$ of $\geq 2.5 \mathrm{mM}$ was used for the geriatric resuscitation protocol based on existing literature [23-25]. A receiver operator characteristic curve analysis was performed in our population, and the results of this analysis support our definition of elevated VL: the optimal cut-off was found to be $>2.4 \mathrm{mM}$, with a sensitivity of $52.6 \%$ and a specificity of $79.5 \%$ for mortality.

Our results demonstrate that standard physiologic variables of SBP and HR may not identify increased risk

Table 3 Stepwise multivariate logistic regression analysis: Predictors of in-hospital mortality

\begin{tabular}{|c|c|c|c|}
\hline Variable & Odds ratio & $95 \% \mathrm{Cl}$ & $P$ value \\
\hline \multicolumn{4}{|c|}{ Model 1: Lactate $\left(r^{2}=0.29 ; \mathrm{H}-\mathrm{L}=0.87\right)$} \\
\hline $\mathrm{VL} \geq 2.5 \mathrm{mM}$ & 2.62 & $1.49-4.58$ & $<0.001$ \\
\hline Glasgow coma scale 3-12 & 11.49 & $6.20-21.30$ & $<0.001$ \\
\hline Injury severity score $\geq 16$ & 3.40 & $1.86-6.21$ & $<0.001$ \\
\hline Age $\geq 85$ years & 2.10 & $1.17-3.77$ & 0.01 \\
\hline \multicolumn{4}{|c|}{ Model 2: Occult hypoperfusion* $\left(r^{2}=0.28 ; H-L=0.55\right)$} \\
\hline Occult hypoperfusion* & 2.12 & $1.20-3.77$ & 0.01 \\
\hline Glasgow coma scale 3-12 & 12.28 & $6.63-22.74$ & $<0.001$ \\
\hline Injury severity score $\geq 16$ & 3.47 & $1.90-6.36$ & $<0.001$ \\
\hline Age $\geq 85$ years & 2.02 & $1.13-3.61$ & 0.02 \\
\hline \multicolumn{4}{|c|}{ Model 2: TVS $\left(r^{2}=0.28 ; H-L=0.77\right)$} \\
\hline Abnormal TVS† & 1.71 & $0.74-3.93$ & 0.21 \\
\hline Glasgow coma scale 3-12 & 14.97 & $8.52-26.29$ & $<0.001$ \\
\hline Injury severity score $\geq 16$ & 3.88 & $2.23-6.76$ & $<0.001$ \\
\hline Age $\geq 85$ years & 1.92 & $1.11-3.32$ & 0.02 \\
\hline \multicolumn{4}{|c|}{ Model 3: Shock index $\left(r^{2}=0.27 ; H-L=0.69\right)$} \\
\hline Shock index $\geq 1$ & 1.18 & $0.39-3.58$ & 0.78 \\
\hline Glasgow coma scale 3-12 & 15.38 & $8.69-27.23$ & $<0.001$ \\
\hline Injury severity score $\geq 16$ & 3.86 & $2.19-6.81$ & $<0.001$ \\
\hline Age $\geq 85$ years & 1.85 & $1.07-3.22$ & 0.03 \\
\hline \multicolumn{4}{|c|}{ Model 4: Lactate and TVS $\left(r^{2}=0.29 ; H-L=0.72\right)$} \\
\hline $\mathrm{VL} \geq 2.5 \mathrm{mM}$ & 2.58 & $1.47-4.52$ & $<0.001$ \\
\hline Abnormal TVSt & 1.46 & $0.62-3.47$ & 0.39 \\
\hline Glasgow coma scale 3-12 & 11.23 & $6.06-20.83$ & $<0.001$ \\
\hline Injury severity score $\geq 16$ & 3.36 & $1.84-6.13$ & $<0.001$ \\
\hline Age $\geq 85$ years & 2.14 & $1.19-3.84$ & 0.01 \\
\hline
\end{tabular}

Stepwise logistic regression, with entry and exit values set at $p<0.15$ for all models.

Abbreviations: $\mathrm{Cl}$, confidence interval; $\mathrm{VL}$, venous lactate; TVS, traditional vital signs; $\mathrm{H}-\mathrm{L}$, hosmer-lemeshow goodness of fit statistic.

*Occult hypoperfusion: VL $\geq 2.5 \mathrm{mM}$ with normal TVS.

†Abnormal TVS: Systolic blood pressure $<90 \mathrm{~mm} \mathrm{Hg}$ and/or heart rate $>$

120 beats/min. 
of mortality in older trauma patients, while an elevated lactate can be used to identify patients with an increased risk for in-hospital mortality, signifying that an increased risk of mortality may not be recognized in current triage and resuscitation protocols that do not incorporate VL. The geriatric population may be especially susceptible to under-evaluation of risk because of comorbidities, polypharmacy and decreased physiologic reserve. We believe an initial VL drawn in the ED or in a pre-hospital setting may improve the identification of significantly injured geriatric patients. Further, we advocate the implementation of a geriatric-specific resuscitation protocol that includes an initial VL to aid in resuscitation and care protocols.

Several recent studies in non-geriatric trauma patients corroborate our findings that lactate is more strongly associated with mortality following trauma than standard physiologic variables [14,30,31]. Meregalli et al. examined 44 high-risk, hemodynamically stable trauma patients, and showed that nonsurvivors had higher lactate than survivors at 12 hours, 24 hours, and 48 hours, whereas there were no differences in mean arterial pressure, HR, and arterial blood oxygenation at any time between survivors and nonsurvivors [31]. On receiver operator characteristic curve analysis, the area under the curve was greater for lactate than for mean arterial pressure, $\mathrm{HR}$, arterial bicarbonate, $\mathrm{PaO}_{2} / \mathrm{FiO}_{2}$, and urine output for predicting mortality. Vandromme et al. studied trauma patients in whom hemodynamic measures were indeterminate (SBP between $90-110 \mathrm{~mm} \mathrm{Hg}$ ), and demonstrated that lactate was a significantly better predictor of mortality than pre-hospital SBP and ED SBP [14]. Lavery and colleagues compared VL $\geq 2 \mathrm{mM}$ against standard triage criteria in their ability to identify major injury, and showed that VL was significantly better in predicting ISS, intensive care unit resource utilization, and hospital length of stay [30].

Additionally, two studies in elderly trauma patients have shown that standard physiologic variables may not identify severe injury $[3,6]$ : Lehmann et al. studied nearly 14000 geriatric trauma patients, and showed that HR and SBP were not predictive of severe injury [6], while Zarzuar and colleagues demonstrated that only in geriatric patients was age $\mathrm{x}$ SI a better predictor of mortality than HR, SBP, and SI alone [3].

A recent systematic review reported that initial lactate is useful for risk assessment, and an elevated lactate requires close monitoring for signs of deterioration [15]; yet few guidelines incorporate an initial lactate measurement [17-19]. Based on the results of this study, VL measurements should be used in the ED for patients for whom no activation occurs based on TVS. Further, we recommend that future studies examine the value of adding a point-of-care measurement of VL for either pre-hospital activation or delayed activation in the ED. While both venous and arterial lactate measurements have been shown to be markers of serious injury following trauma, VL eliminates the need for arterial sampling and correlates strongly with arterial lactate $[15,30]$.

There are several potential limitations to our study: First, the study evaluated mortality in our geriatric trauma population, who sustained predominantly minor blunt injuries from fall and motor vehicle mechanisms of injury, which limits the ability to generalize our findings for trauma populations unlike our own. However, we believe the majority of elderly trauma patients seen at other institutions present with similar injury characteristics [12]. Second, compliance with VL measurements was $75.39 \%$. Patients without a VL closely resembled patients with a $\mathrm{VL}<2.5 \mathrm{mM}$ for mortality and probability of survival; therefore, we believe the association between elevated VL and mortality would remain had all patients received a VL measurement. Third, due to the retrospective design we do not have all potentially relevant data to compare our studies to the existing literature, including emergent interventions in the ED, pre-injury location (e.g. home, nursing facility) and withdrawal of support. Fourth, there are additional activation and resuscitation parameters that we did not compare VL with, such as revised trauma score, GCS, and respiratory rate. Our study was intended to compare predictive ability of physical signs of cHI (SBP and HR) and metabolic indicators of $\mathrm{CHI}$ (VL) for mortality. Other institutions use base deficit as a metabolic marker of $\mathrm{cHI}$; we did not examine base deficit because our geriatric resuscitation protocol specifies routine collection of VL.

\section{Conclusions}

These results add to the growing body of literature demonstrating that $\mathrm{VL}$ is a predictor of mortality following trauma, and that standard physiologic variables of SBP and HR are not independent predictors of mortality in older trauma patients. These findings were demonstrated in a high volume level I trauma center with a large percentage of elderly trauma patients. Determining initial VL levels may improve the identification of $\mathrm{cHI}$, resuscitative efforts, and outcome of geriatric patients, particularly those with unrecognized hypoperfusion.

\section{Abbreviations}

TVS: Traditional vital signs; SBP: Systolic blood pressure; HR: Heart rate; CHI: Circulatory hemodynamic instability; SI: Shock index; VL: Venous lactate; OH: Occult hypoperfusion; ED: Emergency department; GCS: Glasgow coma score; ISS: Injury severity score; OR: Odds ratio; Cl: Confidence interval.

\section{Competing interest}

The authors declare that they have no competing interests. 


\section{Authors' contributions}

$\mathrm{KS}$ and $\mathrm{DBO}$ are responsible for the integrity of the data in this study and the accuracy of the data analysis. KS, PO and DBO were involved in writing the manuscript. KS and PB were involved in data selection and data collection. KS and PO conceived the study idea. CM and PB reviewed the manuscript for accuracy. All authors read and approved the final manuscript.

\section{Author's information}

St. Anthony Hospital, Trauma Research Department, Denver, CO (Mrs. Kristin Salottolo and Dr. David Bar-Or); St. Anthony Hospital, Trauma Services Department, Denver, CO (Drs. Mains and Offner and Mrs. Pamela Bourg); Swedish Medical Center, Trauma Research Department, Englewood, CO (Mrs. Kristin Salottolo and Dr. David Bar-Or); Rocky Vista University, Parker, CO (Drs. Bar-Or and Mains).

\section{Financial support}

Support provided by St. Anthony Hospital, Lakewood, CO.

All the authors state that they have no financial or other conflicts of interest to disclose.

This study was presented as a poster presentation at the 2011 Annual meeting for the American Association for the Surgery of Trauma (AAST) in Chicago, IL.

\section{Author details}

'Trauma Research Department, St. Anthony Hospital, 11600 W. 2nd Place, Lakewood, CO 80228, USA. ${ }^{2}$ Trauma Research Department, Swedish Medical Center, Englewood, CO 80113, USA. ${ }^{3}$ Trauma Services Department, St. Anthony Hospital, Lakewood, CO 80228, USA. ${ }^{4}$ Rocky Vista University, Aurora, CO 80011, USA.

Received: 6 November 2012 Accepted: 30 January 2013 Published: 14 February 2013

\section{References}

1. Allgower M, Burri C: ["Shock index"]. Dtsch Med Wochenschr 1967 92:1947-1950

2. Paladino L, Subramanian RA, Nabors S, Sinert R: The utility of shock index in differentiating major from minor injury. Eur J Emerg Med 2011, 18:94-98.

3. Zarzaur BL, Croce MA, Magnotti LJ, Fabian TC: Identifying life-threatening shock in the older injured patient: an analysis of the National Trauma Data Bank. J Trauma 2010, 68:1134-1138.

4. Scalea TM, Simon HM, Duncan AO, Atweh NA, Sclafani SJ, Phillips TF, Shaftan GW: Geriatric blunt multiple trauma: improved survival with early invasive monitoring. J Trauma 1990, 30:129-134. discussion 134-126.

5. Victorino GP, Chong TJ, Pal JD: Trauma in the elderly patient. Arch Surg 2003, 138:1093-1098.

6. Lehmann R, Beekley A, Casey L, Salim A, Martin M: The impact of advanced age on trauma triage decisions and outcomes: a statewide analysis. Am J Surg 2009, 197:571-574. discussion 574-575.

7. Martin JT, Alkhoury F, O'Connor JA, Kyriakides TC, Bonadies JA: 'Normal' vital signs belie occult hypoperfusion in geriatric trauma patients. Am Surg 2010, 76:65-69.

8. Rogers A, Rogers F, Bradburn E, Krasne M, Lee J, Wu D, Edavettal M, Horst M: Old and undertriaged: a lethal combination. Am Surg 2012, 78:711-715.

9. Eastridge BJ, Salinas J, McManus JG, Blackburn L, Bugler EM, Cooke WH, Convertino VA, Wade CE, Holcomb JB: Hypotension begins at $110 \mathrm{~mm} \mathrm{Hg}$ : redefining "hypotension" with data. J Trauma 2007, 63:291-297. discussion 297-299.

10. Jansen TC, van Bommel J, Mulder PG, Rommes JH, Schieveld SJ, Bakker J: The prognostic value of blood lactate levels relative to that of vital signs in the pre-hospital setting: a pilot study. Crit Care 2008, 12:R160.

11. Paladino L, Sinert R, Wallace D, Anderson T, Yadav K, Zehtabchi S: The utility of base deficit and arterial lactate in differentiating major from minor injury in trauma patients with normal vital signs. Resuscitation 2008, 77:363-368.

12. Pudelek B: Geriatric trauma: special needs for a special population. AACN Clin Issues 2002, 13:61-72.

13. Schulman AM, Claridge JA, Young JS: Young versus old: factors affecting mortality after blunt traumatic injury. Am Surg 2002, 68:942-947. discussion 947-948.
14. Vandromme MJ, Griffin RL, Weinberg JA, Rue LW 3rd, Kerby JD: Lactate is a better predictor than systolic blood pressure for determining blood requirement and mortality: could prehospital measures improve trauma triage? J Am Coll Surg 2010, 210:861-867. 867-869.

15. Kruse O, Grunnet N, Barfod C: Blood lactate as a predictor for in-hospital mortality in patients admitted acutely to hospital: a systematic review. Scand J Trauma Resusc Emerg Med 2011, 19:74.

16. Neville AL, Nemtsev D, Manasrah R, Bricker SD, Putnam BA: Mortality risk stratification in elderly trauma patients based on initial arterial lactate and base deficit levels. Am Surg 2011, 77:1337-1341.

17. Antonelli M, Levy M, Andrews PJ, Chastre J, Hudson LD, Manthous C, Meduri GU, Moreno RP, Putensen C, Stewart T, Torres A: Hemodynamic monitoring in shock and implications for management. International Consensus Conference, Paris, France, 27-28 April 2006. Intensive Care Med 2007, 33:575-590.

18. Bradburn E, Rogers FB, Krasne M, Rogers A, Horst MA, Belan MJ, Miller JA: High-risk geriatric protocol: Improving mortality in the elderly. J Trauma Acute Care Surg 2012, 73:435-440.

19. Jacobs DG, Plaisier BR, Barie PS, Hammond JS, Holevar MR, Sinclair KE, Scalea TM, Wahl W: Practice management guidelines for geriatric trauma: the EAST Practice Management Guidelines Work Group. J Trauma 2003, 54:391-416.

20. Bourg P, Richey M, Salottolo K, Mains CW: Development of a geriatric resuscitation protocol, utilization compliance, and outcomes. J Trauma Nurs 2012, 19:50-56.

21. Federal Interagency Forum on Aging Related Statistics: Older Americans 2000: Key Indicators of Well-Being: National Center for Health Statistics; 2000 http://www.agingstats.gov/Main_Site/Data/2000_Documents/entire_report. pdf. Access date: January 3, 2013.

22. Emergency Medical and Trauma Services Section: Colorado Trauma Registry Inclusion/Exclusion Criteria:: Colorado Department of Public Health and Environment; 2011. http://www.colorado.gov/cs/Satellite?blobcol=urldata\& blobheadername1=Content-Disposition\&blobheadername2=ContentType\&blobheadervalue $1=$ inline $\% 3 B+$ filename $\% 3 D \% 22$ Registry+Manual +Section+A+-+Inclusion+Exclusion.pdf\%22\&blobheadervalue2=application\% 2Fpdf\&blobkey=id\&blobtable=MungoBlobs\&blobwhere $=1251848990095$ \&ssbinary=true. Access date: January 3, 2013

23. Callaway DW, Shapiro NI, Donnino MW, Baker C, Rosen CL: Serum lactate and base deficit as predictors of mortality in normotensive elderly blunt trauma patients. J Trauma 2009, 66:1040-1044.

24. Howell MD, Donnino M, Clardy P, Talmor D, Shapiro NI: Occult hypoperfusion and mortality in patients with suspected infection. Intensive Care Med 2007, 33:1892-1899.

25. Shapiro NI, Howell MD, Talmor D, Nathanson LA, Lisbon A, Wolfe RE, Weiss JW: Serum lactate as a predictor of mortality in emergency department patients with infection. Ann Emerg Med 2005, 45:524-528.

26. Phillips S, Rond PC 3rd, Kelly SM, Swartz PD: The failure of triage criteria to identify geriatric patients with trauma: results from the Florida Trauma Triage Study. J Trauma 1996, 40:278-283.

27. Blow O, Magliore L, Claridge JA, Butler K, Young JS: The golden hour and the silver day: detection and correction of occult hypoperfusion within 24 hours improves outcome from major trauma. J Trauma 1999, 47:964-969.

28. Claridge JA, Crabtree TD, Pelletier SJ, Butler K, Sawyer RG, Young JS: Persistent occult hypoperfusion is associated with a significant increase in infection rate and mortality in major trauma patients. J Trauma 2000, 48:8-14. discussion 14-15.

29. Thom O, Taylor DM, Wolfe RE, Myles P, Krum H, Wolfe R: Pilot study of the prevalence, outcomes and detection of occult hypoperfusion in trauma patients. Emerg Med J 2010, 27:470-472.

30. Lavery RF, Livingston DH, Tortella BJ, Sambol JT, Slomovitz BM, Siegel JH: The utility of venous lactate to triage injured patients in the trauma center. J Am Coll Surg 2000, 190:656-664.

31. Meregalli A, Oliveira RP, Friedman G: Occult hypoperfusion is associated with increased mortality in hemodynamically stable, high-risk, surgical patients. Crit Care 2004, 8:R60-R65.

doi:10.1186/1757-7241-21-7

Cite this article as: Salottolo et al:: A retrospective analysis of geriatric trauma patients: venous lactate is a better predictor of mortality than traditional vital signs. Scandinavian Journal of Trauma, Resuscitation and Emergency Medicine 2013 21:7. 